\title{
Serum Angiopoietin-2 as marker of plasma leakage in Dengue viral infection
}

\author{
Novie Homenta Rampengan ${ }^{1,}$, , Dasril Daud², Sarah Warouw ${ }^{1}$, Idham Jaya Ganda ${ }^{2}$ \\ ${ }^{1}$ Department of Child Health, Medical Faculty of Sam Ratulangi University, Manado, North Sulawesi, Indonesia \\ ${ }^{2}$ Department of Child Health, Medical Faculty of Hasanuddin University, Makassar, South Sulawesi, Indonesia
}

\section{Email address}

novierampengan@yahoo.com (N. H. Rampengan), drdasril@gmail.com (D. Daud), sarahwarouw2002@yahoo.com (S. Warouw), dhamjaya_spa@yahoo.co.id (I. J. Ganda)

\section{To cite this article:}

Novie Homenta Rampengan, Dasril Daud, Sarah Warouw, Idham Jaya Ganda. Serum Angiopoietin-2 as Marker of Plasma Leakage in Dengue Viral Infection. American Journal of Clinical and Experimental Medicine. Vol. 3, No. 1, 2015, pp. 39-43.

doi: 10.11648/j.ajcem.20150301.15

\begin{abstract}
Background. The immunopathology of DHF/DSS is yet completely understood. Angiopoietin-2 (Ang-2) produced by endothelial cell could promote vascular leakage by disrupting endothelial junction. Few studies reported the role of Ang-2 in dengue viral infection. Objective. To study the role of Ang-2 in promoting vascular leakage in dengue viral infection. Methods. Prospective cohort study conducted at Prof. Dr. R.D. Kandou, Pancaran Kasih and R.W. Mongonsidi General Hospitals in Manado, from March 2013 to October 2014. Children, aged 1-15 years old, with the diagnosis of dengue viral infection according to 1997 World Health Organization (WHO) criteria [Dengue Fever (DF), Dengue Hemorrhagic Fever (DHF), Dengue Shock Syndrome (DSS)], were recruited consecutively. Written informed consent was obtained from parents or legal guardian. Blood samples were obtained at the time of diagnosis and 48 hours after, evaluated for complete blood counts, albumin, NS-1, Ig M, Ig G anti-dengue, and Ang-2. Results. During the study period, there were 119 children with dengue viral infections (39 DF, 39 DHF, 41 DSS). Patient's characteristics: female predominance, age between 1.5-8.5 years old, well nourished, and mean albumin level were less than $\leq 3.5 \mathrm{gr} \%$ in the DHF and DSS group. Ang-2 level at admission were 2,486.21 pg/dl, 3,194.95 pg/dl and 4,005.32 pg/dl in DF, DHF and DSS group, respectively. Anova test analyses shows significant differences in serum Ang-2 level at admission between DF, DHF and DSS group $(\mathrm{p}<0.0001)$. Between those groups, DSS group had the highest level of Ang-2, followed with DHF and DF. Discussion. Mean albumin level were 3.88 gr\%, 3.39 gr\% and $2.63 \mathrm{gr} \%$ in DF, DHF and DSS group, respectively. This results shows increasing plasma leakage with increasing severity of dengue viral infection. Increasing level of Ang-2 can promote blood vessels instability therefore causing increased vascular permeability and subsequent plasma leakage. In this study, we found significant elevation of serum Ang-2 in patiens with DSS compared to DHF and DF but the difference was not significant between DF and DHF group. Conclusions. Angiopoietin-2 contributes in transient systemic vascular leak in dengue viral infection.
\end{abstract}

Keywords: Dengue Viral Infection, DF, DHF, DSS, Angiopoietin-2

\section{Introduction}

Dengue viral infection, especially Dengue Hemorrhagic Fever (DHF), is still emerging cause of significant morbidity and mortality in Tropical and Sub Tropical region. ${ }^{1}$ Its incidence is about 50 millions cases each year with approximately 500,000 cases of DHF and about 24,000 cases died. $^{2}$ The classical course of dengue virus infection ranges from asymptomatic infection to severe disease, known as dengue shock syndrome (DSS). ${ }^{3}$ The latter is characterized by a transient endothelial hyperpermeability, of which the hallmark that distinguish it from dengue fever (DF). ${ }^{4}$ The plasma leakage in DHF usually last no more than 48 hours and followed with early and spontaneously resolution. ${ }^{5}$

The immunopathology of DHF/DSS is still incompletely understood. Endothelial cells play a crucial role in the pathophysiology of plasma leakage in dengue viral infection. ${ }^{6}$ Angiopoietin-1 (Ang-1) and Ang-2 are known to bind Tie-2 receptors in endothelial cell. The binding of Ang-2 to Tie receptor could increased angiogenesis induced by 
Vascular Endothelial Growth Factor (VEGF), induced endothelial cells apoptosis and finally potentiating with Tumor Necrosis Factor- $\alpha$ (TNF- $\alpha)$ in expressing surface adhesion molecules. In animal study, added systemic Ang-2 induced vascular hyper permeability and pulmonary congestion. The role of Ang-2 has been reported by many studies, especially in the pathogenesis of diabetic retinopathy, intrauterine growth retardation, pleural effusion, congestive heart disease, tumors and sepsis. ${ }^{7}$ However, up until now, there are only two studies reported by Michels et al. ${ }^{8}$ and van de Weg et al. ${ }^{9}$ evaluating the role of Ang-2 in promoting vascular leakage associated with dengue viral infection. The purpose of this study is to evaluate the role of Ang-2 in the occurance of vascular leakage associated with dengue viral infection and provide better understanding of the immunopathology of DHF and DSS.

\section{Materials and Method}

A prospective cohort study conducted at pediatric ward of Prof. Dr. R.D. Kandou General Hospital, Pancaran Kasih General Hospital and R.W. Mongonsidi General Hospital in Manado, from March 2013 to October 2014. Children, aged 1-15 years old, with dengue viral infection fulfilling the World Health Organization criteria, 1997, were consecutively recruited. Written informed consent was obtained from the patients parents or legal guardian following full and detail explanation regarding the study's protocol. We excluded children diagnosed with bacterial infection or other viral infection based on physical examination and laboratory findings, malnutrition, obesity and patients receiving corticosteroid treatments. The study protocol was approved by the Ethics' Committee of hospital. All patients with dengue viral infection were further classified into 3 groups according to clinical manifestation and laboratory : DF, DHF (I \&II), and DSS (DHF III\&IV).

At time of admission, subjects's blood specimens were drawn in order to evaluate complete blood counts, albumin, globulin, NS-1, Ig M Ig G anti-dengue, and Ang-2. Clinical information was also recorded (age, gender, nutritional status and diagnosis) at that time. All subjects were treated according to World Health Organization (WHO) protocol. At 48 hours of diagnosis, subjects' blood specimens were drawn again in order to evaluate complete blood count, albumin, globulin and Ang-2.

Venous blood specimens were collected at admission and at 48 hours of diagnosis, and immediately centrifuged at 2,000 rpm for 5 minutes. Serum samples were then removed and stored at -30 degree Celsius until they were used for Ang-2 assays. Ang-2 measurements were made using quantitative sandwich enzyme immunoassay.

Statistical analysis consisted of descriptive and comparative analyses. Descriptive analyses were used in order to analyze the characteristics, laboratory findings, and reported in a distribution table. We used anova test to compare the Ang-2 serum between 3 groups. All analyses were performed using SPSS version 21.0.

\section{Results}

During the study period, there were 134 out of 164 patients with dengue viral infections which fulfilled the inclusion criteria. From 134 patients in follow up, 15 patients dropped out from the study, 7 because of the blood sample can't be tested (lysis), 8 because the second dample cannot be obtained (parental refusal), leaving 119 patients completing the study protocol. There were 39 patients with DF, 39 patients with DHF and 41 patients with DSS (Figure 1).

Demography and clinical characteristics of the patients were shown in table 1 . Most of the patients were female (68/119), age between 1.5-8.5 years old, and well nourished (97/119). The onset of fever before admission is between 1-6 days. Mean albumin level were $3.88 \mathrm{gr} \%, 3.39 \mathrm{gr} \%$ and $2.63 \mathrm{gr} \%$ in DF, DHF and DSS group, respectively. Mean albumin level less than $\leq 3.5$ gram $\%$ was found in DBD and DSS group.

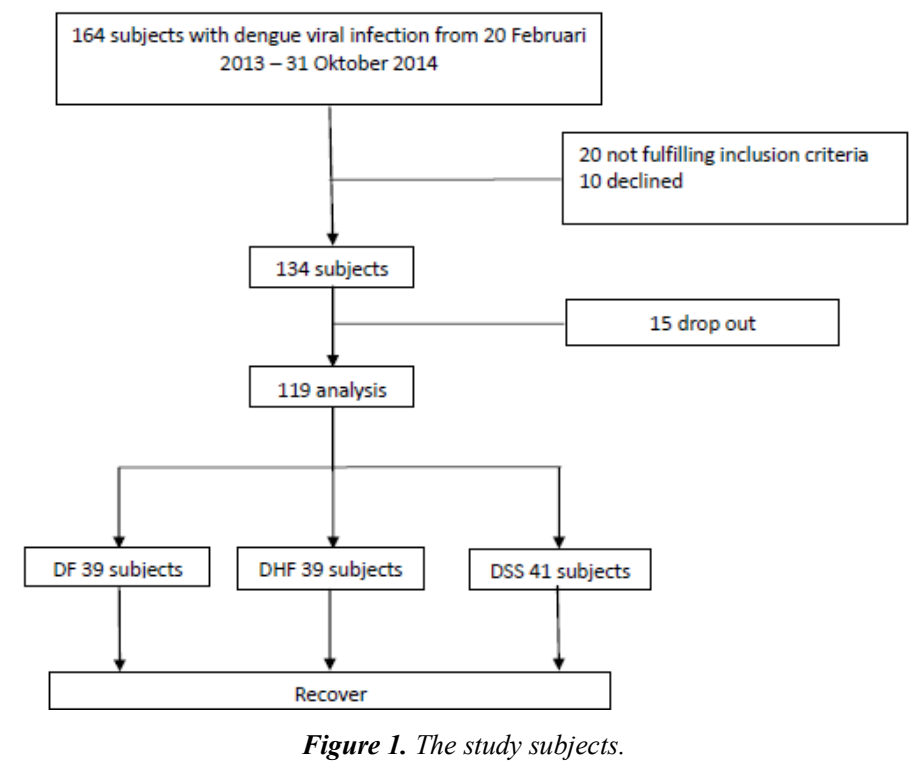


Table 1. Demography and clinical characteristics of the patients studied.

\begin{tabular}{|c|c|c|c|}
\hline Characteristics & DF $(n=39)$ & DHF (n=39) & DSS $(n=41)$ \\
\hline \multicolumn{4}{|l|}{ Gender, n (\%) } \\
\hline Male & $17(43.6)$ & $15(38.5)$ & $19(46.3)$ \\
\hline Female & $22(56.4)$ & $24(61.5)$ & $22(53.7)$ \\
\hline Age, mean (SD), month & $91.87(36.08)$ & $107.49(30.16)$ & $86.35(34.23)$ \\
\hline \multicolumn{4}{|l|}{ Nutritional status, n (\%) } \\
\hline Well nourished & $32(82.1)$ & $32(82.1)$ & $33(80.5)$ \\
\hline Under nourished & $7(17.9)$ & $7(17.9)$ & $8(19.5)$ \\
\hline Onset of fever, days & $2.56(0.60)$ & $4.1(0.80)$ & $4.41(0.67)$ \\
\hline \multicolumn{4}{|l|}{ Laboratory findings } \\
\hline \multicolumn{4}{|l|}{ At admission, mean (SD) } \\
\hline $\mathrm{Hb}, \mathrm{g} / \mathrm{dL}$ & $12.52(1.18)$ & $14.27(1.23)$ & $15.12(1.27)$ \\
\hline $\mathrm{Ht}, \%$ & $36.99(3.43)$ & $42.59(2.99)$ & $45.35(3.44)$ \\
\hline Leucocyte $/ \mathrm{mm}^{3}$ & $3946.1(2392.8)$ & $3556.7(1744.5)$ & $5657.9(2941.2)$ \\
\hline Platelet, $/ \mathrm{mm}^{3}$ & $101128.2(11033.9)$ & $69846.2(20100.5)$ & $48743.9(23790)$ \\
\hline Albumin, g/dL & $3.88(0.41)$ & $3.39(0.51)$ & $2.63(0.54)$ \\
\hline \multicolumn{4}{|c|}{ At 48 hours diagnosed,mean(SD) } \\
\hline $\mathrm{Hb}, \mathrm{g} / \mathrm{dL}$ & $12.53(1.06)$ & $12.21(1.08)$ & $11.88(1.09)$ \\
\hline $\mathrm{Ht}, \%$ & $36.76(3.35)$ & $35.79(2.80)$ & $35.34(2.77)$ \\
\hline Leukocyte, $/ \mathrm{mm}^{3}$ & $6362.3(2513.5)$ & $5291.5(1650.9)$ & $7234.1(2618.2)$ \\
\hline Platelet, $/ \mathrm{mm}^{3}$ & $146179.5(31515.9)$ & $119333(47451)$ & $95488(28167)$ \\
\hline Albumin, $g / d L$ & $3.89(0.48)$ & $3.81(0.4)$ & $3.51(0.54)$ \\
\hline
\end{tabular}

Anova test analyses revealed that there were significant differences serum Ang-2 at admission between DF, DHF and
DSS, with $\mathrm{P}<0.0001$. Between those groups, DSS had the highest level of Ang-2, followed with DHF and DF (Table 2).

Tabel 2. The comparison of serum Ang-2 at admission and at 48 hours diagnosed between DF, DHF and SSD

\begin{tabular}{|c|c|c|c|}
\hline & DF $(n=39)$ & DHF $(n=39)$ & DSS $(n=41)$ \\
\hline Serum level Ang-2 at admission Mean (SD), pg/mL & $2486.21(1534.55)^{*}$ & $3194.95(1572.02)^{*}$ & $4005.32(1706.43)^{*}$ \\
\hline Serum level Ang-2 at 48 hours diagnosed Mean (SD), pg/mL & $2406.84(1243.90)$ & $2115.36(1033.25)$ & $2988.01(1412.48)$ \\
\hline
\end{tabular}

Anova test, ${ }^{*}, \mathrm{p}<0.000$ between three groups at admission

Benferroni test, , $\mathrm{p}<0.000$ between DF and DSS at admission

\section{Discussion}

Dengue viral infection is an arthropod borne virus transmitted through mosquito bites to human. Clinical manifestation vary widely, most commonly in the form of mild self limiting disease known as dengue fever to less common life threatening disease characterized with increased vascular permeability with varying degree, homeostatic disorder, and thrombocytopenia (DHF and DSS). Vascular leakage which is the hallmark of DHF shows endothelial disruption. The increase vascular permeability and subsequent plasma leakage could cause hypovolemic shock knows as DSS. ${ }^{10}$

Many questions still unanswered regarding the exact mechanism of plasma leakage in DHF. Although the elevation of many inflammatory mediator reported in DHF, the increase are only relative compared to dengue fever or healthy subjects. Transient plasma leakage found in DHF without evidence of endothelial injury support the role of circulating mediator of inflammation in promoting vascular hyperpermiability. ${ }^{3}$

In this study we measured albumin level and the results shows mean albumin level were $3.88 \mathrm{gr} \%, 3.39 \mathrm{gr} \%$ and $2.63 \mathrm{gr} \%$ in DF, DHF and DSS group, respectively. Mean level of albumin were $<3.5 \mathrm{gr} \%$ in DHF/DSS group and lower in the DSS group. This shows increasing plasma leakage with increasing severity of dengue viral infection The classification of DF, DHF and DSS in this study was based on clinical manifestation and laboratory (hematocryte). Increased vascular permeability can cause fluid, electrolyte and protein leakage. The use of albumin as a marker of plasma leakage in dengue viral infection was reported by Kalayanarooj, declaring albumin serum level $\leq 3,5 \mathrm{gr} \%$ or changes in albumin level $>0,5 \mathrm{gr} \%$ is consistent with the degree of plasma leakage. ${ }^{11}$

Vascular hyperpermeability and endothelial dysfunction in dengue viral infection was suggested to influence the balance of Ang-1 and Ang-2. Angiopoietin binds tyrosine kinase with immunoglobulin-like loop and epidermal growth factor homology domain (Tie) which expressed in vascular. There are 2 classes of Tie: Tie-1 and Tie-2. During embriogenic development, endothelial cells expressed Tie-1 and Tie-2 receptors. Tie- 2 also expressed in adult endothelial cell. Tie receptor which is well known as Ang-2 binding is Tie-2. ${ }^{12}$

Binding of the agonist Ang-1 to the endothelial Tie-2 receptor via phophatydilinositol-3-kinase (PI3K) Akt pathway promotes vessel stability by recruiting pericytes to nascent blood vessels and preserving cell-cell contacts. Angiopoietin-1 may also have anti-inflammatory action by signaling the down regulation of surface adhesion molecules 
such as Vascular Cell Adhesion Molecule-1 (VCAM-1) and E-selectin. Angiopoietin-1, expressed in supra physiologic concentrations, appear to functions as an anti-permeability agents. $^{13,14}$ In other hand, the action of Ang-2 is more complex, its binding to Tie-2 receptor can inhibits the signaling of Ang-1/Tie-2 and promotes the instability of blood vessels. Interestingly, by using higher concentration or prolonged incubation times with Ang-2 led to the phosphorylation of Tie-2 receptors on some types of endothelial cell and transfected fibroblasts. ${ }^{13-15}$

Angiopoietin-1 is produced by perivascular, smooth muscle cells, and also increased in platelet. Therefore, platelet has a role in maintains the stability of blood vessels. ${ }^{8}$ Angiopoietin-2 is synthesized by endothelial cells and stored in Weibel Palade Bodies (WPB). It quickly released in the circulation upon activation. ${ }^{9}$ Steady state Ang-2 messengerRNA levels increase after endothelial cells exposure to cytokines pro-inflammatory, hypoxia, angiotensin II and thrombin. ${ }^{16}$ Although macrophage do not contain WPB, CD14 containing monocyte could differentiate mimicking endothelial cell and WPB like structure. This activation process of monocyte could increase the secretion of Ang-2. ${ }^{17}$

The release of Ang-2 from WPB occurs upon stimuli of cytokine, thrombin, platelet and activated leukocyte, changes in blood circulation or tissue oxygenation. ${ }^{18}$ Invitro studies reported that without the existence of Ang-2 in the circulation, TNF- $\alpha$ levels $\geq 40 \mathrm{pg} / \mathrm{ml}$ is required to activate endothelial cells. At Ang-2 level $2000 \mathrm{pg} / \mathrm{ml}$ then only $5 \mathrm{pg} / \mathrm{ml}$ or more of TNF- $\alpha$ is required. Angiopoietin-2 could promote the effect of other cytokines in activating endothelial cell, in other words, primes the endothelial cell to the effect of other cytokines. ${ }^{19}$

Angiopoietin-2 is rapidly released into the circulation of humans after a single intravenous injection of lipopolysaccharide $(4 \mathrm{mg} / \mathrm{kb})$, reaching peak levels 4,5 hours after injection and following a profile similar to TNF- $\alpha$. These characteristics suggest that Ang-2 may be a useful early biomarker of early systemic inflammation and endothelial cells activation that contributes to systemic organ injury, dysfunction, and/or failure. ${ }^{20}$

Orfanos et al. reported that serum Ang-2 was mildly increased in patients with SIRS and sepsis but showed a significant increase in severe sepsis. Angiopoietin-2 was also reported inverse correlated with serum albumin $(\mathrm{r}=-0.28 ; \mathrm{p}$ $<0.005)$. This phenomenon might reflect an Ang-2 contribution to the systemic vascular leak, generalized edema formation and organ dysfunction during severe sepsis; the role of Ang-2 in Systemic Inflammatory Response Syndrome (SIRS) and sepsis patients, whose pathologies are usually milder and more localized, may be minimal. ${ }^{16}$

In this study, we found significant elevation of serum Ang2 in patients with DSS at admission compared to DHF and DF. Patients with DSS and DHF had higher serum Ang-2 at admission compared to patients with DF but the difference was only significant between DSS and DF, not between DHF and DF. Serum level Ang-2 in patient with DSS was significantly higher compared to patients with DHF. After 48 hour, the level of Ang-2 decrease significantly in all groups. These findings might reflect the role of Ang-2 in vascular leakage in dengue viral infection.

Our findings is consistent with van de Weg et al who reported that serum levels of Ang-2 increase significantly in patients with dengue viral infection with plasma leakage compared to without plasma leakage $(\mathrm{P}<0.001)$. When patients were classified according to the 2009 WHO dengue case classification, significant elevation of Ang-2 level occur in patients with warning signs compared to without warning signs $(\mathrm{P}=0.004){ }^{9}$

Mitchels et al reported that serum level of Ang-1 in patient with DHF and DSS at enrollment were lower than at discharged and compared with healthy controls. In contrast, patients with DHF and DSS had higher serum level of Ang-2 at enrollment than at discharged and compared with healthy controls. Although the release of Ang-2 from WPB in dengue are unknown, it suggests as a results of an endothelial cells activation by cytokine pro-inflammatory, direct interaction of dengue virus with endothelial cells, release of mast cells products and procoagulant factors such as thrombin. Angiopoietin-2 was also reported correlated inversely with serum albumin $(\mathrm{r}=0.38 ; \mathrm{p}=0.009)$, but it did not correlate significantly with hematocrit value. ${ }^{8}$

The mechanisms of Ang-2 in endothelial barrier disruption were reported by Parikh et al by in invitro study by using human umbilical vein endothelial cell (HUVEC). Human umbilical vein endothelial cell was added with recombinant Ang-2 and stained for F-actin and VE-cadherin. Addition of high Ang-2 serum induced thick actin stress fibers and intercellular gap formation. Endothelial barrier disruption occurred via Rho kinase and myosin light chain kinase activation. ${ }^{13}$ Ong et al also reported the elevated serum level Ang-2 and decreased of serum level Ang-1 in HUVEC with dengue virus exposure. Addition of recombinant Ang-2 in non-toxic dose in HUVEC caused decreasing trans-epithelial electric resistance reading and down regulation of VEcadherin and zona occludin-1 (ZO-1). ${ }^{21}$

The role of Ang-2 in plasma leakage was also reported in acute pancreatitis. Elevated serum Ang-2 levels on admission were associated with plasma leakage, early detection of this biomarker could theoretically identify patients who would best respond to the targeted intervention to stabilize endothelial cells and address pathologic shifts in body fluids. ${ }^{20}$

Limitation of this study is that we only measured the level of Ang-2 in conjunction with albumin levels and clinical manifestation to assets severity of plasma leakage and therefore could not exclude the role of other factors such as TNF- $\alpha$, VEGF, and Ang-1 alone or in combination with Ang2 in promoting vascular leakage.

In this study, we concluded that Ang-2 contributes in transient systemic vascular leak in dengue viral infection (DHF, DSS). It may serve as an early marker of plasma leakage in dengue viral infection and can be targeted as future therapy direction. Further study is needed to evaluate other potential mechanism of vascular leakage in dengue viral infection. 


\section{References}

[1] Kurane I. Dengue hemorrhagic fever with special emphasis on immunopathogenesis. Comp Immunol Microbiol Infect Dis 2007; 30:329-40.

[2] Dewi BE, Takasaki T, Kurane I. Peripheral blood mononuclear cells increase the permeability of dengue virusinfected endothelial cells in association with downregulation of vascular endothelial cadherin. J Gen Virol 2008; 89:642-52.

[3] Hottz E, Tolley ND, Zimmerman GA, Weyrich AS, Bozza FA. Platelets in dengue infection. DDMEC doi: 10.1016/j.ddmec.2011.09.001

[4] Bandyopadhyay S, Lum LCS, Kroeger A. Classifying dengue: a review of the difficulties in using the WHO cases classification for dengue hemorrhagic fever. Trop Med Int Health 2006; 8:1238-55

[5] Srikiatkhachorn A, Ajariyakhajorn C, Endy TP, Kalayanarooj S, Libraty DH, Green S, et al. Virus-induced decline in soluble vascular endothelial growth receptor 2 is associated with plasma leakage in dengue hemorrhagic fever. J Virol 2007; 81:1592-600.

[6] Mitchel M, Djamiatun K, Faradz SMH, Koenders MMJF, de Mast Q, Van der Ven AJAM. High plasma mid regional proandrenomedulin levels in children with severe dengue virus infections. J Clin Vir 2011; 50:8-12.

[7] Siner JM, Bhandari V, Engle KM, Elias JA, Siegel MD. Elevated serum angiopoietin 2 levels are associated with increased mortality in sepsis. Shock 2009; 31:348-53.

[8] Mitchel M, van der Ven AJAM, Djamiatun K, Fijnheer R, de Groot P, Griffioen AW, et al. Imbalance of Angiopoietin 1 and Angiopoietin-2 in severe dengue and relationship with thrombocytopenia, endothelial activation and vascular stability. Am J Trop Hyg 2012; 87(5):943-6.

[9] Van de Weg CAM, Pannuti CS, Van den Ham HJ, de Araujo ESA, Boas LSV, Felix AC, et al. Serum Angiopoetin-2 and soluble VEGF receptor 2 are surrogate markers for plasma leakage in patients with acute dengue virus infection. J Clin Virol 2014; 60:328-35.

[10] Luplertlop N, Misse D. MMP Cellular responses to dengue infection-induced vascular leakage. J Infect Dis 2012;61:298301.

[11] Kalayanarooj S. Dengue classification: Current WHO vs the newly suggested classification for better clinical application? J Med Assoc Thai 2011; 94:74-84.

[12] Hashimoto $\mathrm{T}$ and Pittet JF. Angiopoetin-2: modulator of vascular permeability in acute lung injury? Plos med 2006; e133:3(3): 294-6.

[13] Parikh SM, Mammoto T, Schultz A, Yuan HT, Christiani D, Karumanchi SA, et al. Excess circulating angiopoietin-2 may contribute to pulmonary vascular leak in sepsis in humans. Plos Med 2006; 3:356-69.

[14] Yuan HT, Khankin EV, Karumanchi A, Parikh SM. Angiopoetin 2 is a partial agonist/antagonist of Tie-2 signaling in the endothelium. Mol Cell Biol 2009; 29: 2011-22.

[15] Roviezzo F, Tsigkos S, Kotanidou A, Bucci M, Brancaleone V, Cirino G, et al. Angiopoietin-2 causes inflammation in vivo by promoting vascular leakage. JPET 2005; 314:738-44.

[16] Orfanos SE, Kotanidou A, Glynos C, Athanasiou C, Tsigkos S, et al. Angiopoietin-2 is increased in severe sepsis: correlation with inflammatory mediators. Crit Care Med 2007; 35(1):199206.

[17] Hubbard NE, Lim D, Mukutmoni M, Cai A, Erickson KL. Expression and regulation of murine macrophage angiopoeitin-2. Cell Immunol 2005; 234:102-9.

[18] Clajus C, Lukasz A, David S, Hertel B, Lichtinghagen R, Parikh SM et al. Angiopoetin-2 is a potential mediator of endhotelial barrier dysfunction following cardiopulmonary bypass. Cytokine. 2012;60:352-9

[19] Fiedler U, Reiss Y, Scharpfenecker M, Grunow V, Koidl S, Thurston G, et al. Angiopoietin-2 sensitizes endothelial cells to TNF-alpha and has a crucial role in the induction of inflammation. Nat Med 2006; 12:235-9.

[20] David S, van Meurs M, Kumpers P. Does low Angiopoietin-1 predict adverse outcome in sepsis?. Crit care 2010; 14:180.

[21] Ong SP, Ng ML, Chu JJ. Differential regulation of angiopoietin-1 and angiopoietin-2 during dengue virus infection of human umbilical vein endothelial cells: implications for endothelial hyperpermeability. Med Microbiol Immunol 2013; 202:437-52. 